\title{
Cardiac cellular reprogramming with transient expression vectors: Less is more
}

\author{
Nicholas D. Andersen, MD
}

\footnotetext{
From the Division of Cardiovascular and Thoracic Surgery, Department of Surgery, Duke University Medical Center, Durham, NC.

Disclosures: Author has nothing to disclose with regard to commercial support.

Received for publication Oct 10, 2016; accepted for publication Oct 11, 2016; available ahead of print Nov 17, 2016.

Address for reprints: Nicholas D. Andersen, MD, Division of Cardiovascular and Thoracic Surgery, Duke University Medical Center, Box 3443, Durham, NC 27710 (E-mail: Nicholas.andersen@ duke.edu).

J Thorac Cardiovasc Surg 2017; 153:340-1

$0022-5223 / \$ 36.00$

Copyright $(2) 2016$ by The American Association for Thoracic Surgery

http://dx.doi.org/10.1016/j.jtcvs.2016.10.024
}

A breakthrough in the field of myocardial regeneration was achieved in 2010 when Ieda and colleagues ${ }^{1}$ from the Gladstone Institute demonstrated that cardiac fibroblasts could be reprogrammed directly into induced cardiomyocyte-like cells (iCMs) through expression of the 3 transcription factors Gata4, Mef2C, and Tbx5 (GMT), which normally function to promote cardiac differentiation during early heart development. Given that fibroblasts account for more than $50 \%$ of cells in the adult heart, the strategy of regenerating functional myocardium from resident fibroblasts near infarct zones appeared attractive and within reach. Practical methods for the translation of these revolutionary laboratory discoveries into real-world therapies for the thousands of patients with ischemic cardiomyopathy, however, have remained elusive (Figure 1).

One critical unresolved issue is the optimal method for delivery of the GMT transcription factor elements to cardiac fibroblasts. Previous studies used delivery by retroviral or lentiviral systems, which leads to chronic expression of the GMT transcription factors by integration of the transgenes into the host cell genome; however, integration and chronic expression of the reprogramming factors may not be desirable for several reasons. First, nonspecific transgene incorporation into the host genome can lead to insertional mutagenesis and oncogenic events according to the location of genomic insertion. This lesson was quickly learned from early human gene therapy trials with retroviruses, in which many patients had leukemia develop as a result of transgene integration. ${ }^{2}$ The second issue is that chronic expression of the reprogramming elements may actually be counterproductive to transdifferentiation. Previous studies of induced pluripotent stem cells have shown that reprogramming factors only need to be present for 8 to 10 days before fibroblasts are transformed into a self-sustaining pluripotent state. ${ }^{3}$ Further, mature cardiomyocytes do not chronically express the developmental GMT gene products, and sustained

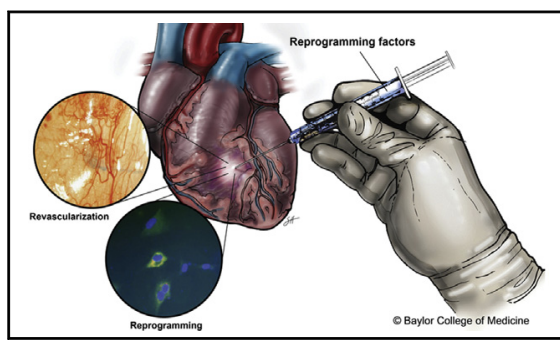

Reprogramming cardiac fibroblasts into induced cardiomyocytes after myocardial infarction.

Central Message

Cardiac cellular reprogramming may be safer and more effective with nonintegrating, transient gene delivery strategies.

See Article page 329

expression of reprogramming elements in reprogrammed cells has even been found to be harmful and to lead to detrimental biologic properties. ${ }^{4}$

In light of these concerns, Mathison and colleagues from Baylor have tested a new strategy, as published in this issue of the Journal, and they achieved cardiac cellular reprogramming in an established mouse infarct model with adenoviral delivery of the GMT reprogramming elements. The purported benefits of adenoviral delivery are that the viral elements do not integrate into the host genome, leading only to transient expression of the transgenes. Mathison and colleagues ${ }^{5}$ found that adenovirally mediated GMT delivery successfully transformed some cardiac fibroblasts into iCMs and was associated with improved ejection fraction at 4 weeks. These results are notable for several reasons. First, transient expression of reprogramming elements was documented to be sufficient to reprogram cardiac fibroblasts into iCMs in a murine model, consistent with previous studies of induced pluripotent stem cells. ${ }^{3}$ Second, the efficacy of reprogramming and the functional performance of the iCMs may actually be enhanced by avoiding the deleterious chronic expression of the reprogramming elements. Finally, the use of adenoviral vectors likely represents a more clinically feasible delivery strategy with fewer mutagenesis concerns than with integrating viral vectors.

There are 2 main limitations of study of Mathison and colleagues. ${ }^{5}$ First, only a small proportion of 




FIGURE 1. Reprogramming cardiac fibroblasts into induced cardiomyocytes after myocardial infarction.

iCMs were produced by these methods, and the long-term functional performance of these cells after 4 weeks is not known. Second, the efficacy of this therapy in human beings remains to be proved, and in the human system immune-mediated destruction and clearance of adenovirally infected cells may be of greater significance than in the murine system. ${ }^{6}$

Despite these unanswered questions, the study by Mathison and colleagues ${ }^{5}$ represents an important step forward. It demonstrates that brief expression of reprogramming factors without genomic integration is a feasible, and arguably superior, approach to myocardial regeneration.

\section{References}

1. Ieda M, Fu JD, Delgado-Olguin P, Vedantham V, Hayashi Y, Bruneau BG, et al Direct reprogramming of fibroblasts into functional cardiomyocytes by defined factors. Cell. 2010;142:375-86.

2. Yi Y, Noh MJ, Lee KH. Current advances in retroviral gene therapy. Curr Gene Ther. 2011;11:218-28.

3. Stadtfeld M, Maherali N, Breault DT, Hochedlinger K. Defining molecular corner stones during fibroblast to iPS cell reprogramming in mouse. Cell Stem Cell. 2008; 2:230-40.

4. Sommer CA, Christodoulou C, Gianotti-Sommer A, Shen SS, Sailaja BS Hezroni $\mathrm{H}$, et al. Residual expression of reprogramming factors affects the transcriptional program and epigenetic signatures of induced pluripotent stem cells. PLoS One. 2012;7:e51711.

5. Mathison M, Singh VP, Chiuchiolo MJ, Sanagasetti D, Mao Y, Patel VB, et al. In situ reprogramming to transdifferentiate fibroblasts into cardiomyocytes using adenoviral vectors: implications for clinical myocardial regeneration. J Thorac Cardiovasc Surg. 2017;153:329-39.e3.

6. Crystal RG. Adenovirus: the first effective in vivo gene delivery vector. Hum Gene Ther. 2014;25:3-11. 